\title{
Patrimoine sectoriel et performances : le cas de l'aquaculture
}

\section{Clarisse Cazals et Audrey Rivaud}

\section{(2) OpenEdition \\ 12 Journals}

Édition électronique

URL : http://journals.openedition.org/ei/5655

DOI : 10.4000/ei.5655

ISSN : 2553-1891

Éditeur

Association Économie et Institutions

Référence électronique

Clarisse Cazals et Audrey Rivaud, "Patrimoine sectoriel et performances : le cas de l'aquaculture », Économie et institutions [En ligne], 20-21 | 2014, mis en ligne le 01 juin 2014, consulté le 19 avril 2019 URL : http://journals.openedition.org/ei/5655; DOI : 10.4000/ei.5655

Ce document a été généré automatiquement le 19 avril 2019

Revue Économie et institutions 


\title{
Patrimoine sectoriel et performances : le cas de l'aquaculture
}

\author{
Clarisse Cazals et Audrey Rivaud
}

\section{Introduction}

1 À partir des années 1990, l'avènement de la notion de développement durable et la remise en cause des indicateurs quantitatifs pour rendre compte du développement d'une communauté humaine, conduisent à une mise en débat de la notion de performance économique (Meadows et al., 1972 ; Sen, 1987 ; Daly, 1991 ; Maric, 1996). Par ailleurs, alors que le processus de mondialisation s'accentue, une partie relativement importante de la littérature traitant de la dynamique des activités économiques s'efforce de mettre en évidence le rôle des "avantages différenciatifs » localisés dans la compétitivité des régions ou des bassins d'activité, (Pecqueur, 2007) dépassant la vision centrée sur les dotations initiales. Le territoire et les activités productives présentes sur ce territoire sont alors envisagés comme deux entités interdépendantes. En partant de ces deux ensembles de critiques, l'objectif de ce travail de recherche est de revisiter la question de la performance dans une optique spatialisée et historiquement construite, à partir de la notion de patrimoine. Cette dernière, tout en étant ancienne et appropriée traditionnellement par les juristes et les économistes dans une logique individuelle, a fait l'objet d'une réflexion renouvelée dans le champ de l'économie institutionnelle à partir de laquelle nous entendons appréhender la performance d'une filière productive dans ses aspects multidimensionnels (Godard, 1990; Barrère et al., 2005 ; Cazals, Sergent, 2009). Plus précisément, en intégrant la diversité des formes patrimoniales et des compromis entre différentes logiques potentiellement contradictoires, l'économie du patrimoine offre la possibilité d'étudier la façon dont les acteurs privés et publics interagissent pour pérenniser une filière en conciliant performances et incertitude productives et commerciales qui sont inhérentes à toute activité économique. 
2 Nous mobilisons ces outils en nous intéressant plus spécifiquement au cas de la filière aquacole européenne qui peut se définir par l'ensemble des activités liées à l'élevage ou à la culture aquatique. Cette filière a donc la particularité de regrouper un grand nombre d'espèces dont la liste exhaustive est délicate à dresser. Toutefois en 2006, la FAO dénombrait 406 espèces aquacoles qu'il est usuel de répartir en trois segments: la conchyliculture, la pisciculture marine et la pisciculture continentale. En dépit de cette diversité structurelle, l'ensemble du secteur est marqué à l'échelle mondiale par un fort développement qui devrait se confirmer dans les prochaines années. En effet, en 2006 la croissance annuelle moyenne de ce secteur était de 6 à $8 \%$, soit la plus élevée de toutes les filières agroalimentaires. Ainsi, l'aquaculture mondiale fournit actuellement près de la moitié des produits de la mer consommés et cette contribution au marché mondial devrait se poursuivre voire s'accentuer dans un futur proche (Hishamunda et al., 2011). Dans ce contexte l'ensemble de l'industrie aquacole connait des mutations aux échelles nationales et européennes, tant sur les plans économiques que politiques, qui conduisent à un repositionnement des acteurs locaux. L'intérêt de questionner la performance dans une perspective élargie se trouve donc renforcée, dans la mesure où les acteurs réalisent des arbitrages non neutres au regard des dimensions patrimoniales de leur activité. Nous faisons ici le choix d'illustrer cette intuition en nous concentrant sur la conchyliculture et plus particulièrement encore sur l'ostréiculture. À cet effet, nous articulons des éléments d'analyse sectorielle et des données collectées dans le cadre d'une enquête sur les stratégies productives des ostréiculteurs arcachonnais, pour prendre en compte l'imbrication de différentes échelles de gouvernement d'une filière et saisir la diversité des performances.

3 Notre propos s'organise de la manière suivante: après avoir présenté les enjeux patrimoniaux de la filière aquacole (1), nous mobilisons les apports de l'économie institutionnelle du patrimoine pour étudier les multiples facettes des performances d'une filière économique (2), nous proposons ensuite de développer cette analyse pour l'ostréiculture arcachonnaise (3) en s'appuyant sur les résultats de notre investigation empirique réalisée à différentes échelles.

\section{Les enjeux patrimoniaux de l'aquaculture : de la prévalence des logiques de préservation}

4 Tout en étant une activité ancestrale reposant sur des pratiques productives traditionnelles dans certaines régions du monde (par exemple en Asie), l'augmentation significative de la production aquacole caractéristique de ces trente dernières années s'est également appuyée sur l'exploitation de rendements d'échelle à partir du modèle économique classique : i) standardisation du mode de production; ii) croissance de la demande ; iii) baisse des coûts de production. Ce schéma a conduit à la mise en place de structures productives innovantes mobilisant de nouvelles technologies dont les impacts négatifs sur les ressources naturelles ont fait l'objet de nombreux travaux scientifiques (Silvert 1992; Piedrahita, 2003; Navarrete-Mier et al. 2010). Pourtant, en dépit des incidences notables en termes de rejets et de risques sanitaires, que cette activité économique peut occasionner sur le milieu naturel, l'aquaculture est perçue comme une alternative crédible au problème de surexploitation des ressources halieutiques et représente une source de protéine animale appréciée par les consommateurs. 
5 Alors que l'expansion économique de l'aquaculture parait incontournable, la filière aquacole européenne ne s'inscrit pas dans cette trajectoire. Malgré un poids économique non négligeable ${ }^{1}$, la production européenne stagne depuis 2008 et ne parvient à satisfaire qu'1/3 de la consommation qui devrait quant à elle continuer à progresser. Même si la question peut se décliner différemment selon les espèces et les pays, la pérennité de cette filière au niveau européen apparaît comme incertaine. Pour approfondir la compréhension des multiples enjeux associés aux formes de développement ou plus modestement au maintien des productions aquacoles dans un contexte de forte pression de la demande, une étude institutionnelle du patrimoine se révèle particulièrement pertinente. Elle permet de produire une lecture multidimensionnelle des performances sectorielles, intégrant les dimensions économiques, environnementales ou encore culturelles associées à la pratique des cultures aquatiques.

6 Nous l'avons tout juste évoqué, l'affirmation d'un nécessaire développement de l'aquaculture est associée aux difficultés croissantes des pêches maritimes et notamment au problème de surexploitation des ressources. Elle est donc concomitante à la mise en place de mesures de protection de ces ressources naturelles à l'échelle européenne et nationale. Néanmoins, le développement de l'aquaculture soulève paradoxalement de nouvelles questions en termes de développement soutenable qui ont impliquées, au niveau macroéconomique la mise en place de différents dispositifs d'action publique ayant pour objectif d'articuler les logiques d'usage (niveau de production) et de préservation (protection de l'environnement). Le tableau 1, présenté ci-dessous, tente de rendre compte de ces dispositifs qui sont révélateurs selon nous, à la fois de la prégnance, mais également du caractère multidimensionnel des enjeux patrimoniaux associés au développement de cette filière. 
Tableau 1. Les dispositifs d'action publique encadrant le développement de l'aquaculture

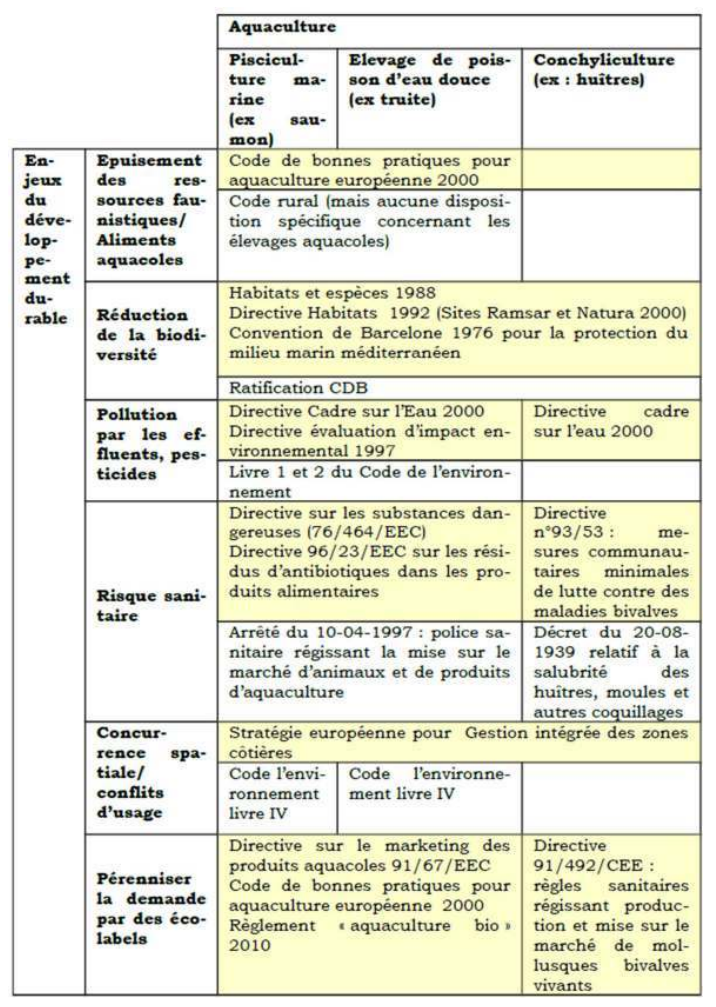

Cellule jaune : Réglementation européenne Cellule blanche : Réglementation française

Source : les auteurs

7 À la lecture de ce tableau, on peut d'abord noter la nature fragmentée de la filière, dans la mesure où les dispositifs sont marqués par des différences selon les espèces. Cependant, une lecture plus englobante permet de souligner également le fait que l'ensemble de cette filière est plus ou moins directement associées à la sauvegarde du patrimoine naturel, ce dernier étant à la fois envisagé comme une contrainte - non dégradation du milieu - mais également comme un facteur - garantie de la qualité des produits - pour le développement de la production. À ce titre les produits conchylicoles constituent un exemple emblématique en étant directement concernés par la législation relative à la qualité du milieu, comme la Directive Cadre sur l'Eau (DCE). En effet, ces produits ont pour principale caractéristique d'être des organismes filtreurs qui accumulent des particules de phytoplancton essentielles à leur croissance, mais aussi du fer, des métaux lourds et diverses pollutions présentes dans leur environnement. La politique de protection des milieux aquatiques représente donc à la fois un cadre contraignant et une opportunité pour le bon déroulement de la production conchylicole. Toutefois, pour les mollusques - comme pour les autres espèces aquacoles - les préoccupations relatives à la qualité dépassent celles de la qualité du milieu pour se focaliser sur la qualité des produits, comme en témoignent les réglementations encadrant strictement le risque sanitaire ou favorisant le développement des écolabels.

8 Outre le patrimoine naturel, ces dispositifs révèlent également l'existence de liens entre la filière aquacole et le patrimoine paysager. L'aquaculture étant une activité consommatrice d'espace, notamment sur les territoires littoraux soumis à d'importantes 
pressions foncières, elle est fortement concurrencée par une variété d'autres usages (productifs, récréatifs, résidentiel) et usagers. En dépit du potentiel de développement que peut représenter ce secteur d'activité pour les espaces littoraux, les différents usagers des zones côtières réputées pour leurs qualités paysagères - avérées ou sublimées - sont souvent réticents vis-à-vis de l'installation d'exploitations aquacoles. Par ailleurs, comme toute activité d'élevage, le segment piscicole de la filière aquacole suppose la maitrise de la technique de l'alimentation animale ${ }^{2}$ et plus précisément la forte dépendance aux ressources halieutiques épuisables. Dès lors l'action publique à l'échelle macroéconomique reste imprégnée de cette ambivalence: d'une part, la stratégie intégrée des zones côtières qui s'inscrit dans la lignée d'un ensemble de recommandations pour le développement de l'aquaculture; d'autre part, le code de l'environnement français qui fixe de nombreuses contraintes à ce développement.

Cette présentation générale des dispositifs d'action publique encadrant l'activité aquacole confirme la prégnance d'enjeux patrimoniaux associés à des objets divers - milieu naturel, paysage - qui impactent nécessairement, de par leur caractère contraignant $a$ minima, les performances de cette filière. Cependant, elle révèle également l'incomplétude de ces dispositifs à l'échelle européenne et nationale et nous incite à adopter une vision élargie du patrimoine pour en saisir les différentes retombées en termes de performance. A cet égard, nous soutenons l'idée que les dimensions patrimoniales de la filière ne touchent pas uniquement à des enjeux en termes de préservation, de conservation, mais également à des enjeux en termes d'usage, de développement (Barrère et al. 2005).

\section{Diversité des performances : l'apport d'une lecture par l'économie du patrimoine}

10 L'analyse de la performance est une préoccupation récurrente de l'histoire de la pensée économique. Cependant, l'enjeu actuel est bien de dépasser la vision économico-centrée basée sur le critère de l'efficience allocative à l'échelle individuelle, pour développer une approche multi-niveaux de la performance plus synthétique et dynamique. Les diverses dimensions sous-jacentes à la notion de patrimoine nous semble offrir des opportunités pour orienter les recherches dans ce sens et notamment renouveler l'analyse des performances d'une filière.

\subsection{Multi-dimensionnalité des performances et des patrimoines}

11 Que ce soit dans le secteur public ou privé, toute organisation doit désormais faire face à un impératif de performance et donc réaliser des activités de reporting pour rendre compte de son activité. Cependant, si la notion de performance est porteuse en son sein de logiques de rationalisation dans la gestion de diverses ressources, la performance est également au cœur d'une vaste littérature allant de la construction d'indicateurs à même de l'évaluer, à l'identification de ses propres frontières. L'étendue des débats est révélatrice des enjeux qui gravitent autour de cette notion. À notre sens, ces débats alimentent la réflexion dans deux directions distinctes mais complémentaires. D'une part, nombre de recherches insistent dans une perspective normative sur le fait que la performance ne doit pas se limiter à des critères d'accumulation du capital, particulièrement dans un contexte où la durabilité imprègne nos modèles de 
développement. D'autre part, un ensemble de travaux s'attache à démontrer dans une perspective positive que la performance n'est pas uniquement construite par le référentiel économique, mais est profondément ancrée dans un contexte institutionnel, culturel, géographique et historique.

12 S'agissant des travaux à portée normative, il convient pour un ensemble d'auteurs (Meadows et al., 1972 ; Sen, 1987 ; Daly, 1991 ; Godard, 2005) de prendre en considération le fait que la performance entendue dans une perspective élargie doit comprendre des critères visant à satisfaire les libertés humaines et la justice sociale et environnementale. L'approche des capabilités développée par A. Sen $(1987$; 1992) a significativement contribué à la redéfinition substantielle de la notion de performance. De façon synthétique, considérant que la référence au bien-être ne relève pas uniquement des seules dispositions économiques, Sen déplace l'attention vers les conditions individuelles d'atteinte du bien-être. Dès lors, il révèle l'influence de deux faits essentiels : la liberté des choix et les caractéristiques individuelles, afin d'envisager non pas les biens eux-mêmes, mais ce que ceux-ci procurent, non pas les revenus, mais ce que ces derniers permettent d'accomplir (Sen, 1992). Dans cette lignée normative, les travaux menés dans le champ de l'économie de l'environnement ou plus précisément dans le champ de l'économie écologique supportent également une acception élargie des critères de performance. En suivant les propos tenus par M. Faber, l'approche économique standard de laquelle découle la définition de la performance centrée sur des critères quantitatifs exprimés en termes d'allocation de ressources, se caractérise par l'absence de prise en compte de trois éléments fondamentaux: "first, the lack of an adequate conceptualisation of nature, second, a failure to handle the question of justice and third, a failure to deal with dynamics of time" (Faber, 2008, p. 2). Or ces éléments sont indispensables pour appréhender la reproduction des systèmes sociaux et économiques à travers le temps.

13 Dans une perspective plus positive, la littérature traitant des logiques de développement territorial nous invite à considérer le caractère situé dans le temps et dans l'espace de la performance d'une activité en insistant sur la diversité des facteurs institutionnels qui y contribuent et sur la dimension procédurale de cette notion. Les travaux auxquels nous faisons référence sont nombreux (Beccatini, 1992; Feldman, Florida, 1994; Courlet, Pecqueur, 1996; Colletis, Pecqueur, 2005; Kébir, 2004; François et al., 2006). Ils s'attachent à souligner la spécificité des ressources, et considèrent que ces ressources sont à la base d'avantages concurrentiels des territoires, déterminants leurs performances ainsi que celles des activités dont ils sont porteurs.

14 Au regard de ces deux ensembles de travaux, la nécessité d'élargir la notion de performance au-delà de la vision économique individuelle de l'efficience allocative est admise, mais la question de ses modalités d'appréhension à l'échelle d'une filière reste ouverte. Ceci notamment en raison des difficultés d'articuler d'une part, les différentes échelles spatiales et temporelles et d'autre part, le niveau sectoriel et individuel. Ces difficultés nous semblent pouvoir être surmontées par l'approche développée par l'économie du patrimoine.

En effet, cette approche conduit non seulement à un élargissement du spectre du patrimoine bien au-delà des monuments historiques et autres objets culturels, mais elle permet aussi de prendre en compte la place stratégique tenue par le patrimoine dans la construction des territoires pour un grand nombre d'acteurs privés et publics impliqués plus ou moins collectivement dans le processus de patrimonialisation (Landel, 2004; Francois et al, 2006). Le patrimoine est alors appréhendé en tant qu'opérateur analytique. 
Ceci nécessite de dépasser l'assimilation réductrice du patrimoine à une forme spécifique de capital ou encore à une approche centrée sur la définition de typologies patrimoniales pertinentes, à partir de la nature de l'objet ou du type du bien économiques - comme tendent à le faire Greffe, (2003); Vecco, (2007); Benahamou (2012). Le patrimoine est plutôt appréhendé comme un ensemble de ressources communes matérielles et immatérielles produites et/ou préservées par des acteurs pour leur dimension identitaire (Barrère et al., 2005 ; Nieddu et al., 2010). Cette posture conduit à étudier la diversité des compromis institutionnels entre différentes logiques complémentaires et opposées (individuelle/collective; usage/préservation) et à intégrer explicitement les enjeux de la double dimension marchande/non marchande des activités économiques - sans accorder de priorité à l'une ou l'autre. La figure 1 ci-dessous tente de présenter de façon schématique cette posture analytique.

Tout en faisant référence à des objets spécifiques et uniques, on comprend donc que le patrimoine ne renvoie pas uniquement à des biens publics dont les niveaux et les formes de la protection sont déterminés a priori. Différents types d'appropriation, voire de non appropriation, sont possibles. Ainsi, tout type de bien peut faire l'objet d'un processus de patrimonialisation qui dépend de la coordination des acteurs privés et publics. Les patrimoines résultent alors d'une appropriation collective construite sous tension, entre d'une part les différentes logiques d'usage et de conservation, et d'autre part les différentes logiques individuelles et collectives. En inscrivant dans le temps et dans l'espace une collectivité, cette forme d'appropriation est source d'identité et réducteur d'incertitude, et ainsi facteur du développement d'activités économiques dans leur diversité (Salais, Storper, 1993). Cette acception du patrimoine laisse entrevoir la capacité d'une telle catégorie analytique à appréhender conjointement les ressorts de l'action individuelle et collective qui sont déterminants dans l'analyse des performances d'une filière, mais elle implique aussi la nécessité de développer une analyse institutionnelle pour mener à bien ce projet. 
Figure 1. L'analyse patrimoniale

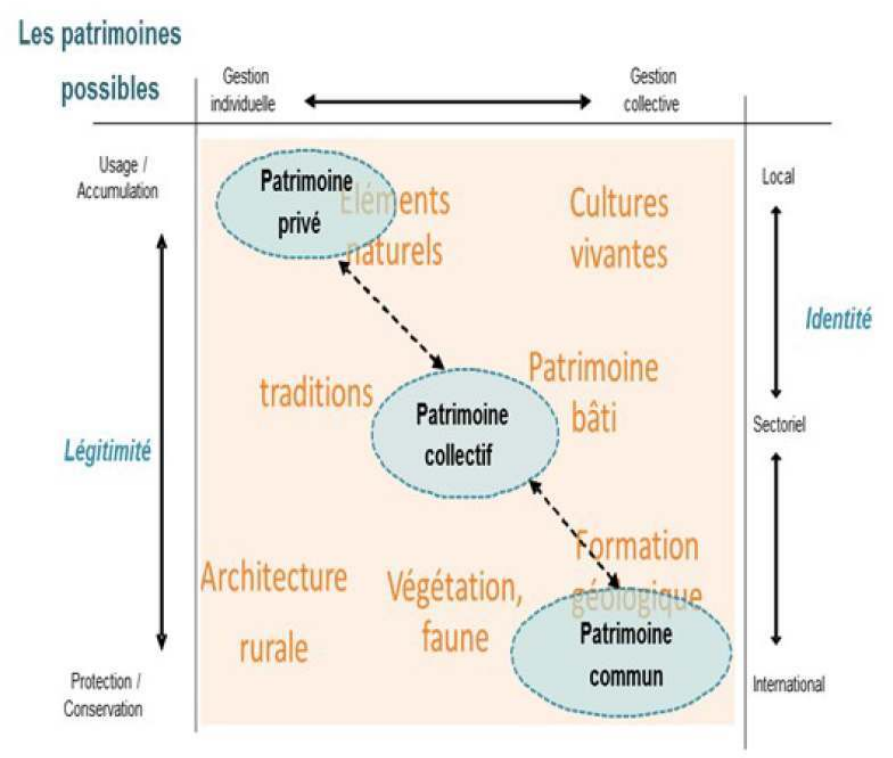

Figure 1 : L'analyse patrimoniale

Processus d'institutionnalisation du patrimoine

Objet support de patrimonialisation

Source : adapté de Cazals ; Sergent, 2009

\subsection{Le patrimoine, une diversité des compromis institutionnels éclairant sur les performances d'une filière}

17 À l'image de l'analyse économique dans son ensemble, la question plus spécifique des performances n'a pas échappé au «tournant institutionnaliste ». À la suite de North (1994) les institutions apparaissent désormais comme des déterminants essentiels des performances économiques, dans la mesure où celles-ci déterminent la structure des interactions. Dès lors, l'approche institutionnelle au sens de North contribue à améliorer notre compréhension de l'histoire économique des États. Cette proposition est aujourd'hui très largement admise (Economides, Egger, 2009) et bien que l'analyse institutionnelle ne fasse pas figure de courant unifié (Théret, 2000), certains auteurs vont jusqu'à considérer que les institutions sont désormais au centre de la théorie économique dominante (Coriat, 1994 ; Jones, Romer, 2009).

Toutefois, s'il est possible d'identifier un paradigme institutionnaliste commun à de nombreux travaux où les institutions peuvent être définies de façon générique comme le résultat d'un collectif (Bessis, 2009; Fossier, Monnet, 2009), le concept d'institution en tant que tel présente des frontières variables qui expliquent la difficulté analytique induite par l'opérationnalisation d'une approche par les institutions. Pour pallier cette difficulté, la mobilisation de la typologie des institutions du capitalisme de Postel (2007), permet de distinguer trois niveaux et formes d'institutions ainsi que leur impact différencié sur l'action. En effet selon l'auteur, le système capitaliste repose sur l'articulation entre une institution macro-sociale fondamentale - la propriété privée -, 
des institutions structurelles qui supportent la première et déterminent le comportement des acteurs, et enfin l'ensemble des règles et conventions communes qui contraignent l'action mais qui sont également façonnées au cours de celle-ci. Cette typologie nécessite alors de prendre en compte les motivations des acteurs et les dimensions éthiques de leur comportement afin de développer une approche complète des institutions (Greif et al., 2009 ; Bessis, 2009 ; Postel 2007).

En combinant la typologie de Postel à l'approche du patrimoine que nous avons développée plus haut, il nous semble possible de développer une analyse de la diversité des mécanismes institutionnels à l'œuvre dans l'économie - gardant à l'esprit que ces mécanismes influencent la performance.

En effet, l'émergence d'un compromis entre des dimensions marchandes et non marchandes potentiellement incompatibles, mais qui au travers de la question patrimoniale sont également envisagées pour leur complémentarité, dépend de la coordination d'acteurs et donc de la réalisation d'un collectif (Bessis, 2009). Ce compromis repose donc sur un ensemble d'institutions formelles et informelles, mais aussi sur des pratiques qui subissent et performent les règles. Les institutions patrimoniales peuvent en effet déterminer la ou les performances d'une entreprise ou d'un secteur, dès lors qu'elles participent à la mise en valeur d'un bien, justifie sa préservation et/ou orientent des évolutions dans les pratiques productives (cf. tableau 2). Finalement, il convient de prendre en compte au-delà des indicateurs traditionnels de vitalité d'une activité ou d'une filière économique (chiffre d'affaire, valeur ajoutée brute, productivité apparente, emplois, etc.), les composantes patrimoniales attachées à cette activité et leur influence sur les pratiques productives à l'échelle des entreprises.

Tableau 2. Typologie des institutions patrimoniales

\begin{tabular}{|c|c|c|c|c|}
\hline & $\begin{array}{ll}\text { Nature } & \text { de } \\
\text { l'institution } & \end{array}$ & $\begin{array}{l}\text { Rapport au } \\
\text { patrimoine }\end{array}$ & $\begin{array}{l}\text { Relations } \\
\text { Actions / } \\
\text { Institutions }\end{array}$ & Exemples \\
\hline $\begin{array}{l}\text { Institutions } \\
\text { fondamentales } \\
\text { (échelle } \\
\text { macrosociale) }\end{array}$ & $\begin{array}{l}\text { Propriété privée/ } \\
\text { absence de } \\
\text { propriété }\end{array}$ & $\begin{array}{l}\text { Nature de l'objet } \\
\text { patrimonial }\end{array}$ & $\mathrm{I} \rightarrow \mathrm{A}$ & $\begin{array}{l}\text { Bien public pur, bien } \\
\text { commun, bien privé }\end{array}$ \\
\hline \multirow[b]{2}{*}{$\begin{array}{l}\text { Institutions } \\
\text { structurelles } \\
\text { (échelle macro } \\
\text { économique) }\end{array}$} & $\begin{array}{l}\text { Formes de } \\
\text { concurrence }\end{array}$ & $\begin{array}{l}\text { Conditions } \\
\text { marchandes } \\
\text { d'usage et } \\
\text { reproduction des } \\
\text { ressources }\end{array}$ & $\mathrm{I} \rightarrow \mathrm{A}$ & $\begin{array}{l}\text { Concurrence } \\
\text { atomistique, } \\
\text { oligopolistique, } \\
\text { monopolistique }\end{array}$ \\
\hline & $\begin{array}{l}\text { Instances } \\
\text { internationales }\end{array}$ & $\begin{array}{l}\text { Conditions } \\
\text { normatives } \\
\text { d'usage et de } \\
\text { reproduction des } \\
\text { ressources }\end{array}$ & $\mathrm{I} \rightarrow \mathrm{A}$ & $\begin{array}{l}\text { Convention de la } \\
\text { biodiversité, } \\
\text { Protocole de Kyoto, } \\
\text { Patrimoine mondiale } \\
\text { de l'Unesco, etc. }\end{array}$ \\
\hline
\end{tabular}




\begin{tabular}{|c|c|c|c|c|}
\hline & $\begin{array}{l}\text { Instances } \\
\text { européennes et } \\
\text { nationales }\end{array}$ & $\begin{array}{l}\text { Conditions } \\
\text { normatives } \\
\text { d'usage et de } \\
\text { reproduction des } \\
\text { ressources }\end{array}$ & $\mathrm{I} \leftrightarrow \mathrm{A}$ & $\begin{array}{l}\text { Directive cadre sur } \\
\text { l'eau, directive } \\
\text { Habitat, etc. }\end{array}$ \\
\hline \multirow{5}{*}{$\begin{array}{l}\text { Institutions } \\
\text { interprétatives : } \\
\text { Règles et } \\
\text { conventions } \\
\text { (échelles méso/ } \\
\text { micro économiques } \\
\text { ) }\end{array}$} & $\begin{array}{l}\text { Instances } \\
\text { régionales }\end{array}$ & $\begin{array}{l}\text { Outils pour } \\
\text { l'usage et la } \\
\text { reproduction des } \\
\text { ressources }\end{array}$ & $\mathrm{I} \leftrightarrow \mathrm{A}$ & Mission d'inventaire, \\
\hline & $\begin{array}{l}\text { Certification des } \\
\text { produits }\end{array}$ & $\begin{array}{lr}\text { Outils pour } \\
\text { l'usage et la } \\
\text { valorsiation des } \\
\text { ressources }\end{array}$ & $\mathrm{I} \leftrightarrow \mathrm{A}$ & $\begin{array}{l}\text { Appellation d'origine } \\
\text { contrôlée, Indication } \\
\text { géographique } \\
\text { protégée, etc. }\end{array}$ \\
\hline & $\begin{array}{lr}\text { Indicateurs } & \text { de } \\
\text { qualité } & \text { des } \\
\text { ressources } & \end{array}$ & $\begin{array}{l}\text { Outils pour } \\
\text { l'usage et la } \\
\text { reproduction des } \\
\text { ressources }\end{array}$ & $\mathrm{I} \leftrightarrow \mathrm{A}$ & $\begin{array}{l}\text { Indicateurs de qualité } \\
\text { de l'eau, de l'air, etc. }\end{array}$ \\
\hline & $\begin{array}{l}\text { Normes de } \\
\text { développement } \\
\text { durable }\end{array}$ & $\begin{array}{lr}\text { Outils } & \text { pour } \\
\text { l'usage, } & \text { la } \\
\text { reproduction et la } \\
\text { valorisation des } \\
\text { ressources }\end{array}$ & $\mathrm{I} \leftrightarrow \mathrm{A}$ & $\begin{array}{l}\text { Guide de bonnes } \\
\text { pratiques, } \\
\text { tourisme, etc }\end{array}$ \\
\hline & Label territorial & $\begin{array}{l}\text { Outils pour } \\
\text { l'usage et la } \\
\text { valorsiation des } \\
\text { ressources }\end{array}$ & $\mathrm{I} \leftrightarrow \mathrm{A}$ & $\begin{array}{l}\text { Parc Naturel Régional, } \\
\text { Grand Site de France, } \\
\text { Ville d'Art et } \\
\text { d'Histoire, etc. }\end{array}$ \\
\hline
\end{tabular}

21 Comme tout exercice de ce style cette typologie n'a pas la prétention d'être exhaustive. Elle permet néanmoins d'insister d'une part sur la prégnance les différents niveaux d'institutions qui formatent les performances des entreprises et des filières économiques, et d'autre part sur le rôle des acteurs en matière de production de règles et de conventions qui peuvent indirectement faire évoluer les institutions structurelles et fondamentales. Il importe donc de se saisir de ces évolutions pour appréhender les performances d'une filière.

Si la première section de ce papier avait pour objectif d'étudier les contours des institutions structurelles en considérant que ces institutions jouent un rôle non négligeable à la fois dans la définition du patrimoine aquacole et sur les performances globales de la filière, nous admettons à l'issue de la présentation de notre cadre conceptuel que ces institutions structurelles ne permettent pas de saisir l'ensemble du processus de patrimonialisation qui caractérise cette filière. Nous avons donc cherché à nous focaliser dans la suite de ce travail sur les institutions interprétatives. 


\section{Du patrimoine à la performance : zoom sur l'ostréiculture}

23 Une des pistes envisageables pour travailler sur les institutions interprétatives, dans le but de saisir avec finesse la diversité des enjeux patrimoniaux et le processus de mise en patrimoine, consiste en la collecte de données individuelles sur les pratiques et les représentations des acteurs de la filière aquacole. C'est la piste que nous avons retenue, dans la mesure où cet angle d'analyse nous permet de confronter effectivement les dimensions marchandes et non-marchandes de l'activité et d'approcher les dynamiques d'hybridation qui en découlent. Compte tenu de l'ampleur de la tâche, mais également des spécificités de chaque sous-filière au sein de l'aquaculture ${ }^{3}$, nous faisons le choix de nous concentrer ici sur une des composantes du secteur aquacole qu'est l'ostréiculture. Tout en ayant conscience des limites que notre choix méthodologique implique en termes de mise en perspective de l'analyse à l'échelle de cette filière fragmentée, celui-ci nous permet toutefois de produire une analyse exploratoire relativement poussée des liens entre patrimoine et performance.

\subsection{Méthode et données}

24 Dans le but de traiter les performances de la filière ostréicole par le prisme des pratiques productives et des représentations patrimoniales, nous avons opéré un nouveau resserrement analytique en nous intéressant au cas de l'ostréiculture dans le bassin d'Arcachon. Nous verrons que le choix de ce territoire se révèle particulièrement pertinent pour alimenter la réflexion sur une approche renouvelée de la performance. Pour tenter de comprendre les compromis productifs et les représentations issus de la confrontation des dynamiques d'usage et de préservation de l'activité nous imbriquons différentes données et différentes échelles d'analyse.

Outre les sources d'empirie nous permettant d'alimenter l'analyse du la filière aquacole à l'échelle européenne, nous nous appuyons sur des données méso-économiques afin d'appréhender le fonctionnement et la dynamique de la filière ostréicole et de saisir les spécificités arcachonnaises. Ces données méso-économiques sont à la fois des données qualitatives issues d'un corpus documentaire et d'entretiens à dire d'expert.

Pour approcher plus directement les logiques d'action et de représentations individuelles, nous mobilisons les données issues d'une enquête par questionnaire soumise en face à face à 117 ostréiculteurs du Bassin d'Arcachon, au cours de l'été 2011. Le questionnaire avait vocation à identifier le positionnement des professionnels quant au caractère multifonctionnel de leur activité d'une part et à décrire les pratiques productives autrement dit les stratégies adoptées en termes de production d'autre part ${ }^{4}$. Ces données ont pu être complétées par des données de structure sur les exploitations (volume de production, chiffre d'affaires, surface d'exploitation, main d'œuvre, etc.) provenant du recensement ostréicole réalisé en 2010 par le Comité Régional de la Conchyliculture Arcachon Aquitaine (CRC-AA). 
Tableau 3. Synthèse des matériaux empiriques mobilisés

\begin{tabular}{|c|c|}
\hline Type d'approche & Sources \\
\hline Analyse macroéconomique & $\begin{array}{l}\text { Rapports FAO } \\
\text { Documents ministériels et de la commission européenne } \\
\text { Données Amadeus } \\
\text { Enquête à dire d'experts auprès des gestionnaires et pro- } \\
\text { fessionnels } \\
\text { Littérature et littérature grise }\end{array}$ \\
\hline \multirow{3}{*}{$\begin{array}{l}\text { Analyse méso-économique } \\
\text { Spécification des pratiques et ar- } \\
\text { ticulation secteur/territoire }\end{array}$} & $\begin{array}{l}\text { CNC / CIPA } \\
\text { Agreste (premier recensement ostréicole 2001) } \\
\text { CER, CRC-AA, Université de Bordeaux (tableau de bord } \\
\text { 2009) }\end{array}$ \\
\hline & $\begin{array}{l}\text { Enquête à dire d'experts auprès des gestionnaires et pro- } \\
\text { fessionnels }\end{array}$ \\
\hline & Littérature et littẻrature grise \\
\hline \multirow{2}{*}{$\begin{array}{l}\text { Analyse micro-économique } \\
\text { Repérage et analyse des pra- } \\
\text { tiques }\end{array}$} & $\begin{array}{l}\begin{array}{l}\text { Données CRC-AA issues du recensement ostréicole } \\
(\mathrm{n}=117) 2010\end{array} \\
\end{array}$ \\
\hline & $\begin{array}{l}\text { Enquête par questionnaire auprès des ostréiculteurs } \\
(\mathrm{n}=117) 2011\end{array}$ \\
\hline
\end{tabular}

Afin d'ordonner l'ensemble des informations contenues dans la base, nous nous appuyons sur une analyse des correspondances multiples (ACM). Ce traitement nous conduit à mettre en évidence l'existence de proximités et de distances entre les pratiques des ostréiculteurs enquêtés, les caractéristiques personnelles et leurs représentations. Les informations collectées au cours de notre enquête et des entretiens à dire d'experts nous permettent par ailleurs d'apporter un certain nombre d'éléments d'ordre qualitatifs qui enrichissent la lecture réalisée par les méthodes de l'analyse factorielle.

\subsection{Des logiques productives aux représentations patrimoniales : des facteurs de la diversité}

\section{L'ostréiculture du bassin d'Arcachon : une activité à pérenniser}

Qu'il s'agisse du volume de production annuel - environ 10000 tonnes - ou du nombre d'exploitations - aux alentours de 300 -, l'ostréiculture arcachonnaise ne représente que $8,5 \%$ de l'ostréiculture nationale (source CNC, 2010).

Figure 2

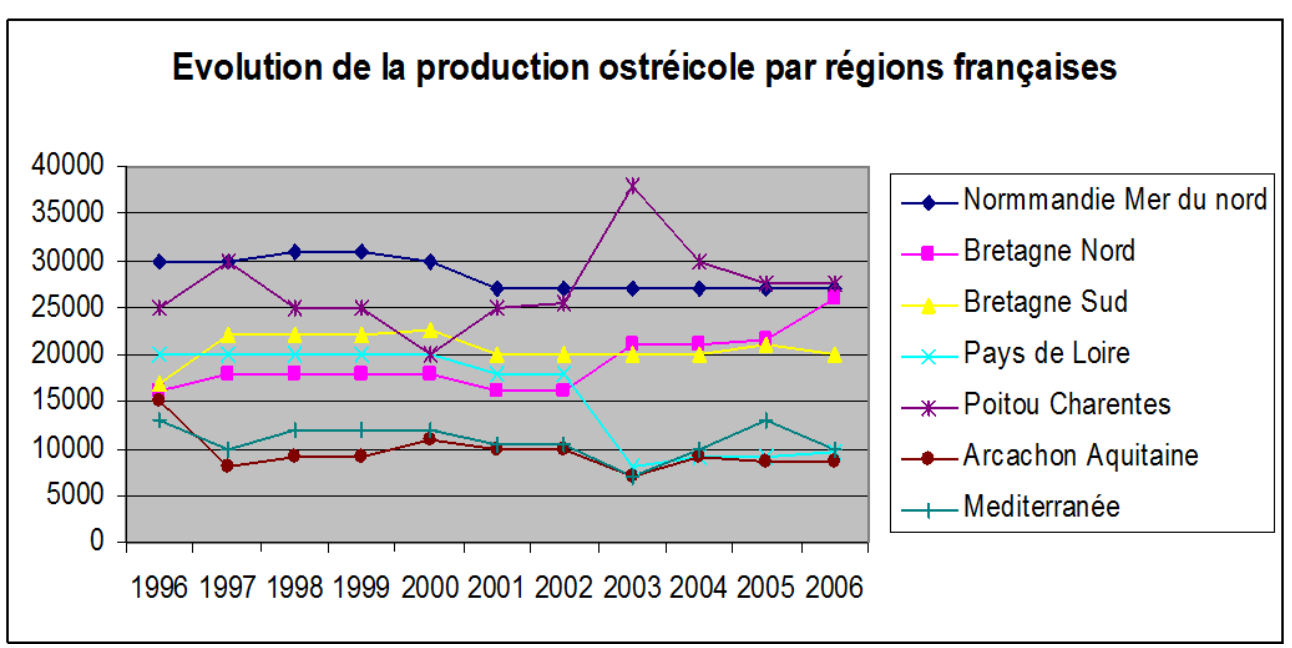

Source : adapté de http://www.cnc-france.com 

$\mathrm{XIX}^{\mathrm{e}}$ siècle. Cette présence historique implique que l'ostréiculture arcachonnaise entretient un lien multiple et complexe à son territoire. Le Bassin d'Arcachon est un espace d'attractivité et d'intérêts écologiques variés qui participe au renom de l'ostréiculture locale. Dans le même temps, cette activité produit un bien traditionnel de qualité symbole d'une certaine image de marque, façonne les paysages et occupe une fonction de sentinelle du milieu en raison de la forte dépendance de l'activité à la qualité des eaux littorales. Pour ces diverses raisons, elle participe en retour à l'identité du Bassin et organise autour d'elle le développement d'activités de loisirs diversifiées allant de la visite du « Musée de l'huître » à la dégustation. L'ostréiculture arcachonnaise constitue à ce titre un vecteur d'attractivité touristique dont le potentiel n'est pas totalement exploité ${ }^{6}$ (SIBA, 2008). Cette référence au développement touristique atteste, dans une certaine mesure, du poids et de la teneur de la demande de patrimoine ostréicole. Le rapport au temps et à l'espace qui caractérise la production ostréicole arcachonnaise audelà de ses dimensions économiques premières représente donc un volet non négligeable dans l'analyse des performances de la filière. Reconnaissant alors l'existence d'un patrimoine collectif, des stratégies visant à protéger/conserver ces spécificités se justifient.

30 Les questions de protection/conservation trouvent en outre une résonnance particulière dans la mesure où le secteur ostréicole européen doit faire face, depuis le milieu des années 2000, à d'importantes perturbations occasionnées par la récurrence des épisodes de mortalités printanières et estivales. À l'issue de ces épisodes répétés, les stocks d'huîtres tendent à se réduire et la production a effectivement diminué de 30 à $40 \%$ entre 2009 et 2010. En réponse à ces événements, les ostréiculteurs envisagent des changements productifs pouvant remettre en cause l'image d'une production traditionnelle véhiculée par la filière sur ce territoire.

31 L'une de ces stratégies, qui introduit une rupture dans les techniques de production et entraine une évolution radicale de l'identité de la filière et du produit, concerne le recours au naissain d'écloserie. Les écloseries, établissements qui parviennent à maitriser en milieu artificiel la reproduction des coquillages, occupent une place grandissante dans le secteur ${ }^{7}$. Ces acteurs ont une influence significative d'une part sur l'évolution des pratiques des ostréiculteurs, d'autre part sur l'évolution des représentations de leur activité. Cette influence est d'autant plus significative que les écloseries mettent à la disposition des producteurs des huîtres stériles - huîtres triploïdes - qui modifient radicalement le cycle de production naturel des coquillages. Si ces huîtres triploïdes permettent aux exploitants ostréicoles d'accroitre leur rentabilité ${ }^{8}$, elles peuvent induire une rupture par rapport aux attentes des consommateurs et restent un objet de controverses au sein de la filière.

\section{Prégnance des logiques productives adaptatives}

Bien que le recours aux huîtres d'écloserie - voire aux huîtres triploïdes - constitue une tendance lourde au sein de la filière ostréicole arcachonnaise, elle n'est pas la seule voie 
possible d'adaptation aux perturbations récentes. Afin de saisir les impacts de différents choix productifs sur l'appréhension des performances de la filière, nous avons étudié précisément les pratiques productives des ostréiculteurs et les représentations leur activité en termes de patrimoine.

Ce travail permet de questionner la diversité des logiques productives et leur stabilité. L'enquête réalisée en partenariat avec Comité Régional de la Conchyliculture Arcachon Aquitaine (CRC-AA) nous conduit à souligner le caractère relativement homogène en termes de performances économiques des exploitations locales et corrobore ainsi la vision d'une ostréiculture traditionnelle dominante supportée par de petites structures familiales. Les résultats d'une Analyse des Correspondances Multiples et d'une Classification Ascendante Hiérarchique réalisées sur les pratiques productives et les caractéristiques des exploitations mettent néanmoins en évidence la coexistence de différentes logiques productives (voir Rivaud, Cazals, 2012). Ces différentes logiques sont qualifiées de : i) logique productiviste, caractéristique d'une intensification des systèmes de production (recours au naissain d'écloserie, utilisation de l'huître triploïde, etc.); ii) logique traditionnelle reposant sur l'exploitation des capacités d'adaptations du milieu (ramassage d'huîtres sur les gisements naturels, par exemple); iii) logiques adaptatives marquées par la diversité des tentatives de réponses aux facteurs de déstabilisation (cf. Tableau 4) pour le détails de ces pratiques productives).

L'existence de trois logiques n'implique pas une égale répartition des acteurs au sein des différentes logiques. L'analyse des données réalisée sur les pratiques productives et les caractéristiques des exploitations invite au contraire à souligner la surreprésentation de la classe associée aux logiques adaptatives avec un effectif de 65 , tandis que les effectifs représentatifs des logiques productivistes et traditionnelles sont respectivement de 27 et 18. La diversité des pratiques mais aussi l'importance des logiques adaptives qui résultent du contexte d'incertitude radicale dans lequel se déroulent les activités productives, remettent en cause l'image de filières économiques reposant sur la coexistence de deux modèles productifs relativement antagoniques (modèle traditionnel versus modèle industriel), pour envisager une structuration autour d'un continuum de stratégies entre ces deux situations polaires. L'intérêt d'avoir identifié ces différentes logiques productives réside dans la mise en dialogue des pratiques et des représentations. Dès lors, nous avons tenté de comprendre dans quelles mesures les choix productifs pouvaient être en résonnance - ou non - avec les images patrimoniales qu'ont les ostréiculteurs de leur activité.

En dépit des difficultés à saisir les représentations et tout en ayant conscience de l'aspect exploratoire de notre approche, nous avons identifié, pour la phase de collecte et de traitement des données, trois composantes des représentations : i) les représentations relatives à l'activité en tant que telle - définition pluridimensionnelle et multifonctionnelle; ii) les représentations sur les moyens de la préservation et de la valorisation de l'activité ; iii) les représentations sur les acteurs de la préservation et de la valorisation. A l'instar de l'analyse des logiques productives, nous mobilisons un traitement des données par une ACM. 
Tableau 4. Les principales pratiques productives ostréicoles sur la Bassin d'Arcachon

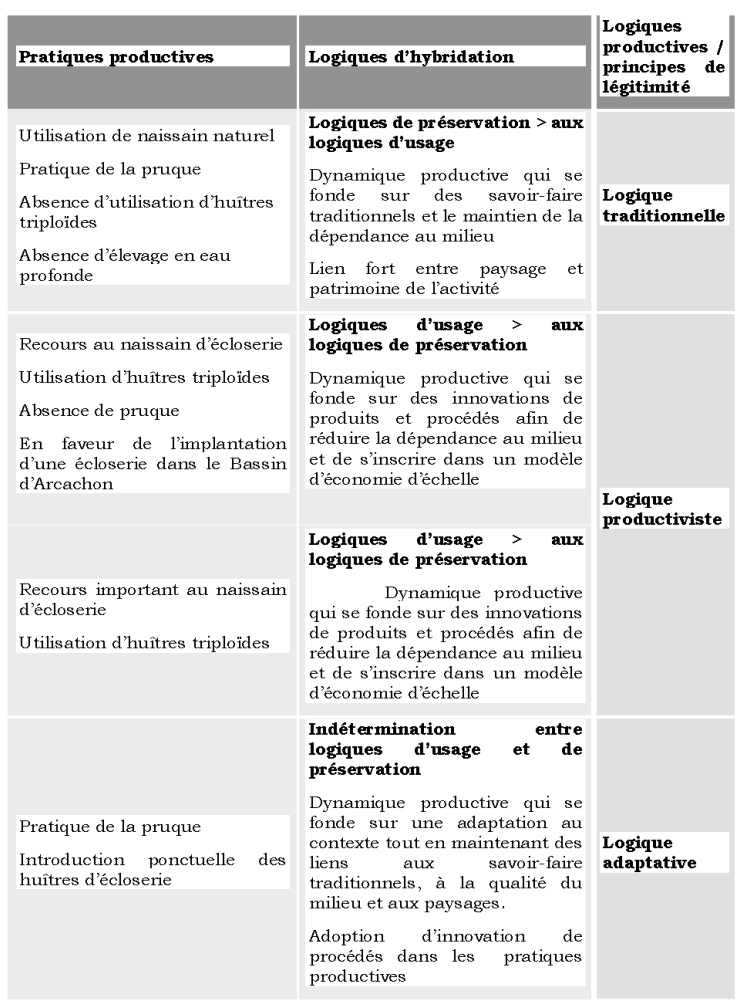

Adapté de Rivaud, Cazals, 2012

Notre objectif étant de mettre en évidence les proximités entre « logiques productives et représentations ", nous nous sommes appuyés sur l'appréhension des représentations présentée juste au-dessus, en trois composantes, afin de structurer l'étude statistique.

La première ACM dont les résultats détaillés sont présentés dans les tableaux 5 et 6 cidessous, permet d'établir des relations entre les logiques productives que nous avons décrites à grand trait et les représentations patrimoniales des ostréiculteurs par rapport à la nature de leur activité. La dialectique usage/conservation propre aux objets patrimoniaux est également introduite à travers la question de l'intérêt de la préservation de l'activité et ses justifications. Les différents résultats mettent en évidence une opposition entre d'une part une représentation de l'ostréiculture en tant qu'activité économique n'ayant pas besoin de faire l'objet de mesure de préservation particulière au sens de "mise sous cloche»-, et d'autre part une vision plus traditionnelle de l'activité. Outre cette opposition entre une ostréiculture économico-centrée et une ostréiculture traditionnelle, les professionnels témoignent également de l'existence de diverses fonctions patrimoniales associées à leur activité. Le traitement des données rend compte d'une vision de l'ostréiculture en tant qu'indicateur de la qualité du milieu intrinsèquement liée au patrimoine naturel, qui doit être préservée en raison des dégradations de l'environnement, mais aussi d'une vision en lien avec la production d'un patrimoine paysager considéré comme un levier du tourisme local et qui doit faire l'objet de mesures de préservation en raison de la présence historique de l'ostréiculture sur le territoire. Il convient de noter que l'analyse de ces investigations ne révèle pas de clivage marquant entre les représentations des ostréiculteurs selon les logiques productives dans lesquelles ils sont inscrits. Ainsi, la représentation de l'ostréiculture en tant qu'indicateur 
de la qualité du milieu est partagée par les ostréiculteurs des logiques productivistes et par les ostréiculteurs des logiques traditionnelles. Cependant, pour ces derniers le patrimoine naturel est associé à une représentation en termes de patrimoine paysager, ce qui n'est pas le cas pour les ostréiculteurs productivistes. S'agissant des ostréiculteurs inscrits dans une logique adaptative, ceux-ci reconnaissent la dimension paysagère du patrimoine ostréicole, mais revendiquent également une vision économique globale de la filière ostréicole en prenant en compte ses potentialités touristiques.

Tableau 5. Représentations relatives à l'ostréiculture (définition pluridimensionnelle et multifonctionnelle) : variables de l'ACM 1

\begin{tabular}{|c|c|c|c|}
\hline $\begin{array}{l}\text { Variables et modalités } \\
\end{array}$ & & & $\%$ \\
\hline \multirow{2}{*}{\multicolumn{4}{|c|}{ 1. Logique productive }} \\
\hline adaptatif & 117 & & $55,5 \%$ \\
\hline productiviste & 117 & 34 & $29 \%$ \\
\hline traditionnelle & 117 & 18 & $15,5 \%$ \\
\hline \multicolumn{4}{|l|}{ 2. Activité économique } \\
\hline activeco_non & 117 & 81 & $69 \%$ \\
\hline activeco_oui & 117 & 36 & $31 \%$ \\
\hline \multicolumn{4}{|l|}{ 3. Activité traditionnelle } \\
\hline activTrad_non & 117 & 31 & $26,5 \%$ \\
\hline activTrad_oui & 117 & 86 & 73,5 \\
\hline \multicolumn{4}{|c|}{ 4. Indicateur de la qualité du milieu naturel } \\
\hline indicNat_non & 117 & 46 & $39 \%$ \\
\hline indicNat_oui & 117 & 71 & $61 \%$ \\
\hline \multicolumn{4}{|c|}{ 5. Activité indissociable du Bassin d'Arcachon } \\
\hline activIndis_non & 117 & 42 & $36 \%$ \\
\hline activIndis_oui & 117 & 75 & $64 \%$ \\
\hline \multicolumn{4}{|l|}{ 6. Patrimoine parsager } \\
\hline patPays_non & 117 & 77 & $66 \%$ \\
\hline patPays_oui & 117 & 40 & $34 \%$ \\
\hline \multicolumn{4}{|l|}{ 7. Levier du tourisme } \\
\hline lev'Tourism_non & 117 & 101 & $86 \%$ \\
\hline levTourism_oui & 117 & 16 & $14 \%$ \\
\hline \multicolumn{4}{|c|}{ 8. Besoin de préservation de l'activité ostréicole } \\
\hline Pres_non & 117 & 10 & $8,5 \%$ \\
\hline Pres_oui & 117 & 107 & $91,5 \%$ \\
\hline \multicolumn{4}{|l|}{ 9. Raison : poids économique } \\
\hline Pres_Poidseco_non & 117 & 101 & $86 \%$ \\
\hline Pres_Poidseco_aui & 117 & 16 & $34 \%$ \\
\hline \multicolumn{4}{|l|}{ 10. Raison : présence historique } \\
\hline Pres_histo_non & 117 & 91 & $78 \%$ \\
\hline Pres_histo_oui & 117 & 26 & $22 \%$ \\
\hline \multicolumn{4}{|c|}{ 11. Raison : Difficultés actuelles de la filière } \\
\hline Pres_diffActiv_non & 117 & 67 & $57 \%$ \\
\hline Pres_DiffActiv_Pres_oui & 117 & 50 & $43 \%$ \\
\hline \multicolumn{4}{|c|}{ 12. Raison : encombrement du plan d'eau } \\
\hline Pres_encombr_non & 117 & 69 & $59 \%$ \\
\hline Pres_encombr_oui & 117 & 48 & $41 \%$ \\
\hline \multicolumn{4}{|c|}{ 13. Raison : dégradation du milieu } \\
\hline Pres_degra_non & 117 & 65 & $55,5 \%$ \\
\hline Pres_degra_oui & 117 & 52 & $44,5 \%$ \\
\hline \multicolumn{4}{|c|}{ 14. Raison : rôle en matière d'aménagement du territoire } \\
\hline Pres_roleAmen_non & 117 & 104 & $89 \%$ \\
\hline Pres_roleAmen_oui & 117 & 13 & $11 \%$ \\
\hline
\end{tabular}


Tableau 6. Modalités contribuant à la formation des quatre premiers axes de l'ACM1

\begin{tabular}{|c|c|c|}
\hline & Coordonnées négatives & Coordonnées positives \\
\hline $\begin{array}{l}\text { Axe } 1 \\
(13,13 \%)\end{array}$ & $\begin{array}{l}\text { Activité economique_non }(10,2) \\
\text { Activité traditionnelle_oui }(6,8)\end{array}$ & $\begin{array}{l}\text { Activité économique_oui }(23,0) \\
\text { Activité_traditionnelle_non }(18,9) \\
\text { Activité indissociable du BA_non } \\
(5,1) \\
\text { Préservation_non }(9,8) \\
\text { Préservation_historique_oui }(5,8)\end{array}$ \\
\hline $\begin{array}{l}\text { Axe } 2 \\
(11,63 \%)\end{array}$ & $\begin{array}{l}\text { Indicateur qualité milieu_non } \\
(16,6) \\
\text { Patrimoine paysager_oui }(8,6) \\
\text { Levier du tourisme_oui }(8,6) \\
\text { Préservation_non }(4,6) \\
\text { Préservation_historique_oui }(7,6) \\
\text { Préservation_dégradation_non } \\
(12,4)\end{array}$ & $\begin{array}{l}\text { Indicateur qualité milieu_oui } \\
(10,8) \\
\text { Patrimoine paysager_non }(4,5) \\
\text { Préservation_dégardation_oui } \\
(15,5)\end{array}$ \\
\hline $\begin{array}{l}\text { Axe } 3 \\
(9,31 \%)\end{array}$ & $\begin{array}{l}\text { Logique traditionnelle (21,9) } \\
\text { Préservation_non }(21,4) \\
\text { Préservation encombrement_non } \\
(6,1)\end{array}$ & $\begin{array}{l}\text { Logique productiviste( } \mathbf{7 , 0}) \\
\text { Préservation_encombrement_oui } \\
(8,8) \\
\text { Préservation_dégradation } \\
\text { milieu_oui }(4,2)\end{array}$ \\
\hline Axe 41 & $\begin{array}{l}\text { Logique adaptative } \mathbf{( 6 , 2 )} \\
\text { Indicateur qualité milieu_non }(7,0) \\
\text { Acticité indissociable du BA_oui } \\
(6,2) \\
\text { Levier tourisme_oui }(9,8) \\
\text { Préservation_poids éco_oui }(4,9) \\
\text { Préservation_difficulté_oui }(3,7) \\
\text { Préservation_encombrement_non } \\
(6,3)\end{array}$ & $\begin{array}{l}\text { Logique traditionnelle } \mathbf{( 6 , 8 )} \\
\text { Indicateur qualité milieu_oui } \\
(4,5) \\
\text { Activité indissociable BA_non } \\
(11,0) \\
\text { Patrimoine paysager_oui }(3,5) \\
\text { Préservation_non }(3,6) \\
\text { Préservation_encombrement_oui } \\
(9,0)\end{array}$ \\
\hline
\end{tabular}

Contributions aux axes entre parenthèses

Ces résultats se révèlent particulièrement intéressants dans la mesure où ils mettent en évidence le fait qu'il est difficile d'identifier une relation stable entre une vision patrimoniale - certes multiple - de l'ostréiculture et des logiques productives spécifiques. En outre, ils témoignent selon nous du fait que les frontières des institutions interprétatives sont régulièrement redéfinies par les acteurs en fonction de l'évolution des problèmes auxquels ils sont confrontés - typiquement par les épisodes de mortalités par exemple.

\section{Acteurs publics, acteurs privés : tous concernés par la protection de l'ostréiculture}

L'intégration des variables «moyens de la préservation et de la valorisation » dans la deuxième étape de l'étude statistique (cf. ACM2 Annexe 1) confirme la distinction ressortie de la première ACM. D'un côté, on retrouve une vision de l'ostréiculture en tant qu'activité traditionnelle s'opposant à tout mode de protection normatif et préférant des modalités de préservation de l'activité ayant trait au maintien et à la transmission des savoir-faire traditionnels. De l'autre côté, on identifie une vision économico-centrée qui envisage la préservation de l'activité par des mesures de communication autour du produit et de la profession, par la mise en place de signe officiel de qualité ou encore par le développement de normes. La prégnance du cadre réglementaire est donc diversement appréciée et attendue.

Lors de la troisième étape de l'étude statistique, les variables relatives aux représentations sur les acteurs de la préservation et de la valorisation de l'activité ostréicole sont plus spécifiquement regardées (cf. ACM3 Annexe 2). À l'inverse des deux 
ACM précédentes, nous notons qu'aucune des trois logiques productives ne contribue à la construction d'un axe. Les pratiques productives sont relativement éloignées des représentations sur le rôle des acteurs. En revanche les ostréiculteurs distinguent nettement le rôle de l'État, qui selon eux doit être responsable de la préservation de la qualité du milieu, et le rôle d'une instance telle que le parc naturel marin du Bassin d'Arcachon qui est perçu à la fois comme un acteur de la valorisation et de la préservation de l'activité ostréicole sur le territoire.

$41 \mathrm{Si}$, au regard des évolutions actuelles de la filière, les professionnels sont quasiment unanimes sur la nécessité de préserver l'ostréiculture, ils ont une représentation très hétérogène de la mise en œuvre de cette nécessité. La préservation peut s'inscrire dans une logique purement marchande, mais aussi se croiser, voire s'hybrider avec les dimensions naturelles du patrimoine. Au-delà du caractère éclaté des conditions de préservation de l'ostréiculture dans le discours des exploitants, la mise en perspective des représentations et des pratiques semble illustrer les tensions entre les logiques marchandes et les logiques non marchandes internes à la filière ostréicole sur le Bassin d'Arcachon. Les représentations des acteurs quant au rôle de leur activité sur le territoire - fonction de témoin de la qualité du milieu naturel qu'occupe l'ostréiculture ou fonction d'aménagement paysager notamment - peuvent être contradictoires avec certaines des stratégies de repositionnement opérées au sein des exploitations - notamment avec la montée en puissance d'acteurs tels que les écloseries. Dès lors, nous soutenons l'idée que le changement de pratiques implique des reconfigurations des institutions patrimoniales qui peuvent aller à l'encontre de la vision qu'ont les professionnels de leur activité.

42 Ainsi la performance d'une entreprise envisagée dans ses simples dimensions économiques ne permet pas de rendre compte des dimensions patrimoniales associées à l'activité. Ces éléments d'analyse sont essentiels dans le cadre de la mise en œuvre des politiques d'aménagement du territoire. Les décideurs publics ont à prendre en considération cette dynamique de tension pour être en mesure de préserver les formes patrimoniales relatives à une filière économique en pleine restructuration, s'ils entendent protéger un certain nombre de dimensions identitaires liées à cette activité emblématique.

\section{Conclusion}

Dans ce travail nous avons eu le projet de proposer une grille d'analyse permettant d'analyser les performances d'un secteur. Pour atteindre cet objectif il est apparu nécessaire d'une part d'aborder la performance dans une perspective élargie et d'autre part de se saisir de la délicate question de l'articulation entre performances sectorielle et performances individuelles. Il nous semble que l'analyse par le prisme de l'approche patrimoniale que nous proposons permet de prendre en compte ces deux contraintes. En effet, en insistant sur la diversité des objets susceptibles d'être reconnus comme patrimoine et des compromis entre logiques marchandes et logiques non marchandes, la question des performances de la filière se déplace du problème de la mesure des résultats économiques vers celui de la prise en compte des différentes composantes de la performance et de leurs déterminants institutionnels. En outre nous avons tenté de développer une analyse institutionnelle complète allant des institutions structurelles aux pratiques et aux représentations qui portent les institutions interprétatives. 
D'une manière relativement exploratoire étant donné le caractère structurellement hétérogène de la filière aquacole, nous avons éprouvé cette grille de lecture en nous focalisant à l'échelle microéconomique sur l'ostréiculture du Bassin d'Arcachon. Si la prégnance des institutions structurelles ne peut être discutée, elle contribue principalement à contraindre les acteurs à reconnaître un patrimoine naturel défini par un dispositif réglementaire le plus souvent subi par les producteurs différenciés selon les espèces. En revanche, ces institutions patrimoniales structurelles ont peu d'impacts sur la préservation d'un patrimoine productif collectif à l'échelle territoriale et donc sur la prise en compte des performances socioéconomiques territoriales d'une activité productive. Sur cet aspect le focus réalisé sur l'ostréiculture du Bassin d'Arcachon met en évidence la coexistence de plusieurs logiques de production diversement justifiées par les ostréiculteurs. L'écart entre pratiques, règles et représentations n'est pas révélateur d'un dysfonctionnement mais plutôt de la réflexivité des acteurs sur les institutions vecteur de changement institutionnel. Si l'on accepte que les enjeux sous-jacents à l'analyse d'une filière ne sont pas de réunir les conditions de mise en œuvre d'une boîte à outils mais plutôt de décrypter la mécanique de tension entre les différentes logiques de préservation et d'usage qui se déploient à différentes échelles individuelles et/ ou collectives, la démarche analytique que nous proposons dans ce travail trouve tout son sens.

\section{BIBLIOGRAPHIE}

Barrère C., Barthélémy D., Nieddu M., Vivien F-D. (éds.), 2005, Réinventer le patrimoine. De la culture à l'économie, une nouvelle pensée du patrimoine ?, Collection Gestion de la culture, L'Harmattan, $337 \mathrm{p}$.

Becattini G., 1992, « Le district marshallien : une notion socio-économique », in Benko G., Lipietz A. (Ed.), Les régions qui gagnent - Districts et réseaux : les nouveaux paradigmes de la géographie économique, Presses Universitaires de France, Paris, pp. 35-55.

Benhamou F., 2012, Economie du patrimoine culturel, Edition La Découverte, Paris.

Bessis F., 2009, «L'institution comme réalisation du collectif », Tracès, 2(17), pp. 73-87. <http:// traces.revues.org/4216>

Cazals C., Sergent A., 2009, « Développement durable te patrimoine productif ligneux : une approche interdisciplinaire appliquée à la filière Bois-Papier en Aquitaine », Colloque ASRDLF, 6-8 juillet, Clermont-Ferrand.

Colletis G., Pecqueur B., 2005, « Révélation de ressources spécifiques et coordination située », Revue Economie et Institutions, $\mathrm{n}^{\circ}$ 6-7 $1^{\mathrm{er}}$ et $2^{\text {nd }}$ semestre, Numéro spécial « Proximités et institutions : nouveaux éclairages ».

Coriat B., 1994, "La théorie de la régulation. Origines, spécificités et perspectives », Futur Antérieur, Numéro Spécial « Ecole de la régulation et critique de la raison économique »,pp 101-152. 
Courlet C. et Pecqueur B.(1996), « Districts industriels, systèmes productifs localisés et développement. ", in Abdelmalki L., Courlet C. (Éds.), Les nouvelles logiques du développement, L'Harmattan, Logiques Économiques, pp. 91-102.

Daly H., 1991, "Ecological Economics and Sustainable Development: from concept to policy", World Bank Environment Department, Divisional Working paper ${ }^{\circ}$ 1991-24, Washington D.C., World Bank.

Economides, G., Egger, P.H., 2009, "The role of institutions in economic outcomes: editorial introduction”, European Journal of Political Economy, n² 25, pp. 277-279.

Faber M., 2008, "How to be an ecological economist", Ecological Economics, n 66, pp 1-7.

Feldman MP. et Florida R., 1994, « The Geographic Sources of Innovation: Technological Infrastructure and Product Innovation In the United States », Annals of the Association of American Geographers, vol. 84, pp. 210-229.

Fossier A., Monnet E., 2009, « Les institutions mode d'emploi », Tracès, 2(17), pp 7-28. <http:// traces.revues.org/4183>

Framian, B. V. (2009). Definition of data collection needs for aquaculture: Part 1. Review of the EU aquaculture sector and results of costs and earnings survey. FISH/2006/15-Lot 6 . See http://ec.europa.eu/fisheries/documentation/studies/data_collection_en.

François H., Hirczak M., Senil N., 2006, « Territoire et patrimoine : la co-construction d'une dynamique et de ses ressources ", Revue d'économie régionale et urbaine, (5), pp 683-700.

Godard O., 1990, « Economie, mode de coordination et système de légitimité : analyse de la catégorie de patrimoine naturel », Revue économique 41(2), mars, pp 215-242.

Godard O., 2005, « Les trois courants complémentaires du champ de l'économie de l'environnement : une lecture systématique », Cahier d'épistémologie, $\mathrm{n}^{\circ} 332$, septembre, 53 p.

Greffe X., 2003, La Valorisation économique du patrimoine, La documentation française, 383 p.

Greif A., Lenormand M., 2009, «Qu'est-ce que l'analyse institutionnelle ? », Tracès, 2(17), pp. 181-210. <http://traces.revues.org/4264>

Hishamunda N., Poulain F., Ridler N., 2011, Analyse prospective du développement de l'aquaculture : la méthode Delphi, FAO Document technique sur les pêches et l'aquaculture, n 521, Rome, FAO, 77 p. Jones, C.I., Romer, P.M., 2009, “The new kaldor facts: ideas, institutions, population, and human capital”, American Economic Journal: Macroeconomics n² 2, pp. 224-245.

Kébir L., 2004, Ressource et développement : une approche institutionnelle et territoriale, Thèse de doctorat en Sciences économiques, réalisée à l'Université de Neuchâtel, Neuchâtel, p. 190.

Landel P.-A., 2004, Invention de patrimoines et construction des territoires, Actes du colloque « Ressource territoriale », Le Pradel, Mirabel, 11 p.

Maric M., 1996, « Egalité et équité, l'enjeu de la liberté. Amartya Sen face à John Rawls et à l'économie normative ", Revue française d'économie, vol. XI, n 3, pp. 95-125.

Meadows D.H., Meadows D.L., Jorgen R., Behrens W.W., 1972, Halte à la croissance ? Enquête du Club de Rome. Rapport sur les limites de la croissance, Paris, Fayard, 314 p.

Navarette-Mier F., Sanz-Lazaro C, Marin A., 2010, “Does bivalve mollusc polyculture reduce marine finfish farming environmental impact”, Aquaculture, vol. 306, Issues 1-4, pp. 101-107.

Nieddu, M., Garnier E. Bliard C., L'émergence d'une chimie doublement verte. Revue d'économie industrielle, 2010, no 4, p. 53-84. 
North D., 1994, "Economic Performance Trough Time”, American Economic Review, vol. 84, n 3, pp. 359-368.

Pecqueur B., 2007, «L'économie territoriale : une autre analyse de la globalisation », L'Economie Politique 2007/1, n 33, pp 41-52.

Piedrahita R.H., 2003, "Reducing the potential environmental impact of tank aquaculture effluents through intensification recirculation", Aquaculture, vol. 226, Issues 1-4, pp. 35-44.

Postel N., 2007, « Hétérodoxie et institution », Revue du MAUSS, 2(30), pp. 83-116.

Rivaud A., Cazals C., 2012, « Pour une vision élargie des performances de la filière ostréicole à partir d'une approche en termes de patrimoine », Développement durable et territoires [En ligne], vol. $3, \mathrm{n}^{\circ} 1$.

Salais R., Storper M., 1993, Les mondes de production : enquête sur l'identité économique de la France, École des Hautes Études en Sciences Sociales, Paris, 467 p.

Sen A., 1987, Éthique et économie et autres essais, PUF, Paris, 1993, tr. Fr. de On Ethic and Economics, Blackweel Publishers, Oxford, 1991, 364 p.

Sen A., 1992, Inequality Reexamined, New-York: Russell Stage Foundation, 14., Harvard University Press, Cambirdge, 207 p.

Silver W., 1992, “Assessing environmental impacts on fishing aquaculture in marine waters", Aquaculture, pp. 67-79.

Théret B., 2000, « Institutions et Institutionnalismes. Vers une convergence intra et interdisciplinaire des conceptions de l'institution?", Recherche et régulation série EM 2000-1, 25 p., www.theorie-regulation.org

Vecco M., 2007, Economie du patrimoine monumental, Economica, Paris, 245 p.

\section{ANNEXES}

\section{Annexe 1. ACM2 : Représentations relatives à l'ostréiculture et moyens de la préservation et de la valorisation de l'activité}


Tableau I : Variables supplémentaires de l'ACM2

Les variables constitutives de l'ACM 2 recouvrent variables de l'ACM 1 auxquelles s'ajoutent les 11 variables actives suivantes

\begin{tabular}{|c|c|c|c|}
\hline Variables et modalités & $\mathbf{N}$ & $N=1$ & $\%$ \\
\hline \multicolumn{4}{|c|}{ 1. Moyen de la préservation : norme } \\
\hline MPres_nome_non & 117 & 71 & $61 \%$ \\
\hline MPres_nome_oui & 117 & 46 & $39 \%$ \\
\hline \multicolumn{4}{|c|}{ 2. Moyen de la préservation : valorisation économique du produit } \\
\hline MPres_valo_non & 117 & 96 & $82 \%$ \\
\hline MPres_valo_oui & 117 & 21 & $18 \%$ \\
\hline \multicolumn{4}{|c|}{ 3. Moyen de la préservation : subvention } \\
\hline MPres_subv_non & 117 & 103 & $88 \%$ \\
\hline MPres_subv_oui & 117 & 14 & $12 \%$ \\
\hline \multicolumn{4}{|c|}{ 4. Moyen de la préservation : transmission des savoir-faire } \\
\hline MPres_tran_non & 117 & 87 & $74 \%$ \\
\hline MPres_tran_oui & 117 & 30 & $26 \%$ \\
\hline \multicolumn{4}{|c|}{ 5. Moyen de la préservation : sensibilisation du public } \\
\hline MPres_sensi_non & 117 & 90 & $77 \%$ \\
\hline MPres_sensi_oui & 117 & 27 & $23 \%$ \\
\hline \multicolumn{4}{|c|}{$\begin{array}{l}\text { 6. Moyen de la préservation : maintien des techniques de produc- } \\
\text { aditionnelles }\end{array}$} \\
\hline MPres_maintTrad_non & 117 & 95 & $81 \%$ \\
\hline MPres_maintTrad_oui & 117 & 22 & $19 \%$ \\
\hline \multicolumn{4}{|c|}{ 7. Moyen de la préservation : gestion concertée } \\
\hline MPres_gestConcert_non & 117 & 87 & $74 \%$ \\
\hline MPres_gestConcert_oui & 117 & 30 & $26 \%$ \\
\hline \multicolumn{4}{|c|}{ 8. Moyen de la valorisation : communication } \\
\hline MValo_Com_non & 117 & 72 & $61,5 \%$ \\
\hline MValo_Com_oui & 117 & 45 & $38,5 \%$ \\
\hline \multicolumn{4}{|c|}{ 9. Moyen de la valorisation : qualité du produit } \\
\hline MValo_Quali_non & 117 & 36 & $31 \%$ \\
\hline MValo_Quali_oui & 117 & 81 & $69 \%$ \\
\hline \multicolumn{4}{|c|}{ 10. Moyen de la valorisation : signe officiel de qualité } \\
\hline MValo_Signof_non & 117 & 80 & $68 \%$ \\
\hline MValo_Signof_oui & 117 & 37 & $32 \%$ \\
\hline \multicolumn{4}{|c|}{ 11. Moyen de la valorisation : mise en place de démarche pédago } \\
\hline $\begin{array}{l}\text { MValo_Ped } \\
\text { MValo Ped }\end{array}$ & 117 & 99 & $85 \%$ \\
\hline
\end{tabular}

Tableau II : Modalités contribuant à la formation des quatre premiers axes de I'ACM2

\begin{tabular}{|c|c|c|}
\hline & Coordomnées négatives & Coordonnées positives \\
\hline $\begin{array}{c}\text { Axe } 1 \\
(8,55 \%)\end{array}$ & $\begin{array}{l}\text { Activité économique_oui }(15,6) \\
\text { Activité_tradtionnelle_non }(11,3) \\
\text { Préservation_non }(8,6)\end{array}$ & $\begin{array}{l}\text { Activité economique_non }(6,9) \\
\text { Activité traditionnelle_oui }(4,1) \\
\text { Préservation_historique_oui }(8,6) \\
\text { Moyen de la } \\
\text { préservation_transmission_oui }(4,2) \\
\text { Moyen de la préservation_moyen } \\
\text { tech. trad_oui }(7,1)\end{array}$ \\
\hline $\begin{array}{c}\text { Axe } 2 \\
(8,36 \%)\end{array}$ & $\begin{array}{l}\text { Indicateur qualité milieu_oui }(4,4) \\
\text { Préservation_dégradation_oui } \\
(7,9) \\
\text { Moyen de la préservation_gestion } \\
\text { concertée_oui }(7,1) \\
\text { Moyen de la } \\
\text { préservation_communication_non } \\
(3,4)\end{array}$ & $\begin{array}{l}\text { Activité traditionnelle_non }(3,1) \\
\text { Indicateur qualité milieu_non }(6,8) \\
\text { Levier_tourisme_oui }(5,4) \\
\text { Préservation activité_non }(6,7) \\
\text { Préservation_dégardation_non }(6,4) \\
\text { Moyen de la valo_communication_oui } \\
(5,5) \\
\text { Moyen de la valo_qualité produit_non } \\
(6,6)\end{array}$ \\
\hline $\begin{array}{c}\text { Axe } 3 \\
(7,01 \%)\end{array}$ & $\begin{array}{l}\text { Logique traditionnelle }(7,8) \\
\text { Activité_indispensable_non }(3,5) \\
\text { Préservation_non }(10,2) \\
\text { Moyen de la } \\
\text { préservation_norme_non }(6,4)\end{array}$ & $\begin{array}{l}\text { Levier du tourisme_oui }(3,6) \\
\text { Moyen de la préservation_norme_oui } \\
(9,8) \\
\text { Moyen de la } \\
\text { présrevation_sensibilisation_oui }(5,0) \\
\text { Moyen de la valo_communication_oui } \\
(4,7) \\
\text { Moyen de la préservation_signe } \\
\text { officiel_oui }(3,3)\end{array}$ \\
\hline $\begin{array}{c}\text { Axe } 4 \\
(6,47 \%)\end{array}$ & $\begin{array}{l}\text { Indicateur qualité milieu_oui }(3,5) \\
\text { Moyen de la } \\
\text { préservation_sensibilisation_oui } \\
(15,5) \\
\text { Moyen de la valo_pédagogie_oui } \\
(12,0)\end{array}$ & $\begin{array}{l}\text { Logique productiviste }(5,0) \\
\text { Préservation rôle aménagement_oui } \\
(9,5) \\
\text { Moyen de la } \\
\text { préservation_transmission_oui }(6,6) \\
\text { Moyen de la } \\
\text { préservation_sensibilisation_non }(4,7)\end{array}$ \\
\hline
\end{tabular}

Contributions aux axes entre parenthèses 


\section{Annexe 2. ACM3 : Représentations relatives à l'ostréiculture et moyens de la préservation et de la valorisation de l'activité}

Tableau I : Variables supplémentaires de l'ACM3 l'ACM 1 auxquelles s'ajoutent les 12 variables actives suivantes:

\begin{tabular}{|c|c|c|c|}
\hline Variables et modalités & $\mathbf{N}$ & $\mathbb{N}=1$ & $\%$ \\
\hline \multicolumn{4}{|c|}{ 1. Acteur de la préservation : Etat } \\
\hline APres_Etat_non & 117 & 66 & $56 \%$ \\
\hline APres_Etat_oui & 117 & 51 & $44 \%$ \\
\hline \multicolumn{4}{|c|}{ 2. Acteur de la préservation : Collectivité territoriale } \\
\hline APres_ColTer_non & 117 & 95 & $81 \%$ \\
\hline APres_ColTer_oui & 117 & 22 & $19 \%$ \\
\hline \multicolumn{4}{|c|}{ 3. Acteur de la préservation : Parc Naturel Marin } \\
\hline APres_PNM_non & 117 & 94 & $80 \%$ \\
\hline APres_PNM_oui & 117 & 23 & $20 \%$ \\
\hline \multicolumn{4}{|c|}{ 4. Acteur de la préservation : Représentants professiontrels } \\
\hline APres_RepProf_non & 117 & 75 & $64 \%$ \\
\hline APres_RepProf_oui & 117 & 42 & $36 \%$ \\
\hline \multicolumn{4}{|c|}{ 5. Acteur de 1a préservation : Association de l'environnement } \\
\hline APres_Asso_non & 117 & 111 & $95 \%$ \\
\hline APres_Asso_oui & 117 & 6 & $5 \%$ \\
\hline \multicolumn{4}{|c|}{ 6. Acteur de la préservation : Ostréicult eurs eux-mêmes } \\
\hline APres_Ostrei_non & 117 & 57 & $49 \%$ \\
\hline APres_Ostrei_oui & 117 & 60 & $51 \%$ \\
\hline \multicolumn{4}{|c|}{ 7. Acteur de la valorisation : Etat } \\
\hline AValo_Etat_non & 117 & 103 & $88 \%$ \\
\hline AValo_Etat_oui & 117 & 14 & $92 \%$ \\
\hline \multicolumn{4}{|c|}{ 8. Acteur de la valorisation : Collectivité Territoriale } \\
\hline AValo_ColTer_non & 117 & 80 & $68 \%$ \\
\hline AValo_ColTer_oui & 117 & 37 & $32 \%$ \\
\hline \multicolumn{4}{|c|}{ 9. Acteur de la valorisation : Parc Naturel Marin } \\
\hline AValo_PNM_non & 117 & 104 & $89 \%$ \\
\hline AValo_PNM_oui & 117 & 13 & $11 \%$ \\
\hline \multicolumn{4}{|c|}{10 Acteur de la valorisation : Représentants professionnels } \\
\hline AValo_RepProf_non & 117 & 72 & $61,5 \%$ \\
\hline AValo_RepProf_oui & 117 & 45 & $38,5 \%$ \\
\hline \multicolumn{4}{|c|}{ 11. Acteur de la valorisation : Professionnels du tourisme } \\
\hline AValo_Tourism_non & 117 & 105 & $90 \%$ \\
\hline AValo_Tourism_oui & 117 & 12 & $10 \%$ \\
\hline \multicolumn{4}{|l|}{ 12. Acteur de la valorisation } \\
\hline AValo_ostrei_non & 117 & 43 & $37 \%$ \\
\hline AValo_ostrei_oui & 117 & 74 & $63 \%$ \\
\hline
\end{tabular}




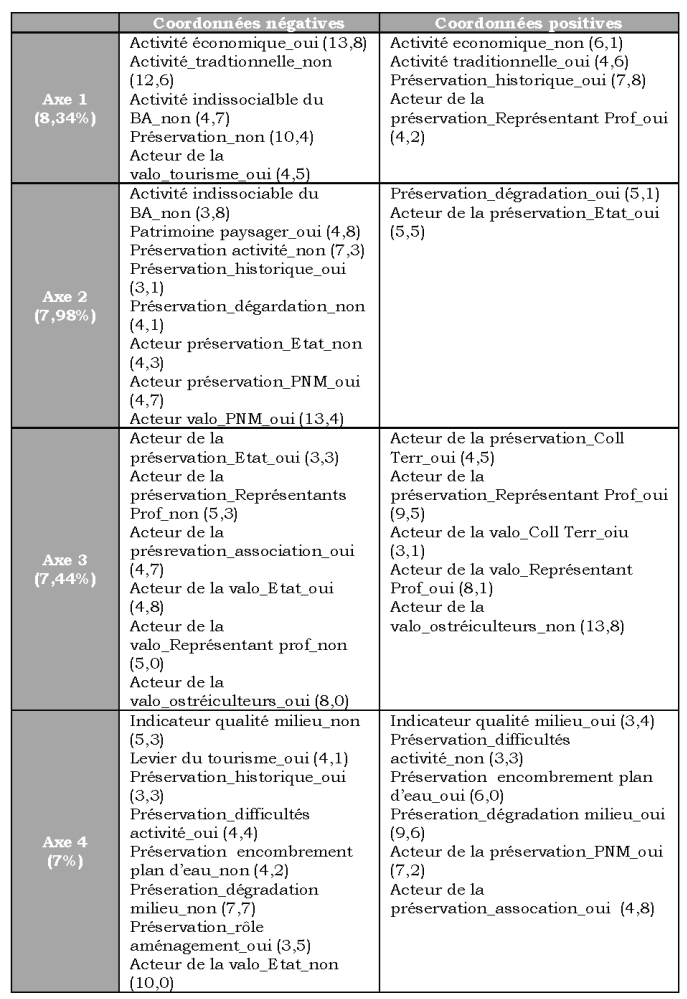

Contributions aux axes entre parenthèses

\section{NOTES}

1. Il est dénombré en 2009, 16500 entreprises, un chiffre d'affaires de 3,5 milliards d'euros et 64000 emplois directs et indirects (Framian, 2009)

2. L'importance de cette dépendance est mesurée par l'indicateur «FIFO» (fish in/fish out) qui évalue de façon littérale, le rapport entre la quantité de poissons sauvages qui entre sous forme de farine dans le cycle de production et la quantité de poissons produite pour la nourriture humaine. Pour les associations environnementalistes ce bilan est très négatif, particulièrement pour les productions intensives de saumon et de crevettes.

3. Pour rappel, l'analyse des institutions structurelles réalisée dans la première section a mis en lumière une nette différenciation entre les filières piscicoles et conchylicoles au sein de l'aquaculture, et a donc révélé l'impossibilité de développer à ce jour un dispositif institutionnel commun à la filière aquacole. Cette situation s'explique notamment par l'existence d'enjeux économiques et environnementaux profondément distincts entre, d'une part des activités productives piscicoles qui doivent faire face à des problématiques assez similaires à celles rencontrées par les filières d'élevage (alimentation, rejets dans le milieu, etc.), et d'autre part les activités conchylicoles qui sont fortement dépendantes de la qualité du milieu, faiblement impactant pour l'environnement.

4. Le questionnaire était constitué de 79 questions, dont 18 questions relatives aux représentations des ostréiculteurs et 61 questions sur les pratiques d'élevage et de commercialisation.

5. Si le nombre d'exploitants ne cesse de baisser, le taux de chargement des parcs est quant à lui à son niveau maximum. 
6. SIBA (2008).

http://www.siba-bassin-arcachon.fr/sites/siba/files/rapport_bva_final.pdf

7. Les écloseries sont présentes dans le secteur ostréicole depuis la fin des années 1990, mais leur poids s'est significativement accru avec les épisodes de mortalités.

8. En effet, la stérilité du coquillage permet de réduire le stress occasionné lors de la période de production et garantit donc une croissance plus rapide. De surcroit, les huîtres triploïdes ne produisant pas de « lait » en période estivale, elles sont plus facilement commercialisables.

\section{RÉSUMÉS}

Si la portée normative et les dimensions multicritères des performances ne sont plus discutées, l'analyse des performances d'une filière reste une question tant pour les acteurs publics que pour les acteurs privés. Pour y faire face nous proposons une analyse en termes de patrimoine. Cela permet d'intégrer explicitement l'horizon temporel et l'ancrage territorial des activités économiques qui tiennent une place centrale dans l'analyse de leurs performances.

En outre le patrimoine reposant sur un compromis entre différentes logiques (individuelle/ collective ; usage/préservation), les contradictions intrinsèques dont est porteuse l'appréhension de la performance ne sont pas éludées. L'application de la grille de lecture patrimoniale à l'étude de la filière aquacole et plus précisément de l'ostréiculture arcachonnaise permet d'en révéler ses potentialités.

If the normative scope and the multi-criteria dimensions of performances are not discussed, the analysis of the performance of an industry remains an issue for private and public actors. To deal with it, this article proposes an analysis in terms of heritage. This makes it possible to integrate time dimensions and territorial anchorage of economic activities which are very important elements in analysis of their performance. Considering that the heritage is based on a compromise between, sometimes contradictory types of logic (individual/collective; use/ preservation), contradictions of performance are taken into account their studies. The heuristic power of the reading of the performance in terms of heritage is assessed by the study of aquaculture industry and more precisely the oyster farming in the Arcachon Bay (France).

\section{INDEX}

Mots-clés : performance, patrimoine, hybridation des dimensions marchandes et non marchandes, stratégies productives, ostréiculture

Keywords : performance, heritage, articulation between market and non market dimensions, productive strategies, oyster-farming

Code JEL B52 - Institutional • Evolutionary, L52 - Industrial Policy • Sectoral Planning Methods, Q22 - Fishery $•$ Aquaculture 


\section{AUTEURS}

\section{CLARISSE CAZALS}

clarisse.cazals[at]irstea.fr

\section{AUDREY RIVAUD}

audrey.rivaud[at]univ-montp3.fr 